\title{
Renal medullary carcinoma and its association with sickle cell trait: a case report and literature review
}

\author{
P. Holland BScPharm, ${ }^{*}$ J. Merrimen $\mathrm{MD}_{1}^{\dagger}$ C. Pringle $\mathrm{MD}^{\ddagger}$ and L.A. Wood $\mathrm{MD}^{\S \|}$
}

\begin{abstract}
Renal medullary carcinoma (RMC) is a rare and aggressive renal malignancy that usually presents at an advanced stage, has a poor prognosis, and is associated with sickle cell trait. We present a case of RMC including radiologic and pathology findings, treatment, and outcome. A review of the literature is also presented, with an emphasis on the association of RMC with sickle cell trait, which was an unknown diagnosis in our patient preoperatively.
\end{abstract}

Key Words Renal medullary carcinoma, sickle cell disease, kidney cancer

Curr Oncol. 2020 February;27(1):e53-e56

www.current-oncology.com

\section{INTRODUCTION}

Renal medullary carcinoma (RMC) is a rare and aggressive cancer of the kidney that primarily affects adolescents and young adults with sickle cell trait (SCT). It was first described by Davis et al. in $1995^{1}$. We report a patient with localized RMC treated with nephrectomy and adjuvant chemotherapy who had a much better outcome than most patients with this diagnosis.

\section{CASE DESCRIPTION}

A 47-year-old man presented with gross hematuria and a 2-week history of right lower back pain. There were no significant abnormal physical exam findings. On past medical history, he stated that he was an ex-smoker. There was no family history of urothelial or renal cancer.

Urinalysis showed 200 red blood cells per microlitre. Hematuria-protocol computed tomography (СТ) imaging identified a $5 \mathrm{~cm}$ moderately enhancing homogenous mass originating from the lower half of the right kidney. The margins were somewhat ill-defined, with extension into the renal collecting system (Figure 1), but no venous extension and no calcification or macroscopic fat content. Small enhancing local lymph nodes were also identified. Subsequent chest radiography and chest Ст showed no evidence of metastases.

The patient underwent cystoscopy, ureteroscopy, and insertion of a right ureteric stent. Urine cytology was negative. The clinical impression was that this was an upper-tract urothelial carcinoma.

Biopsy showed an invasive carcinoma growing in cords, with occasional tubules noted in a background of marked desmoplasia and accompanying neutrophil-rich

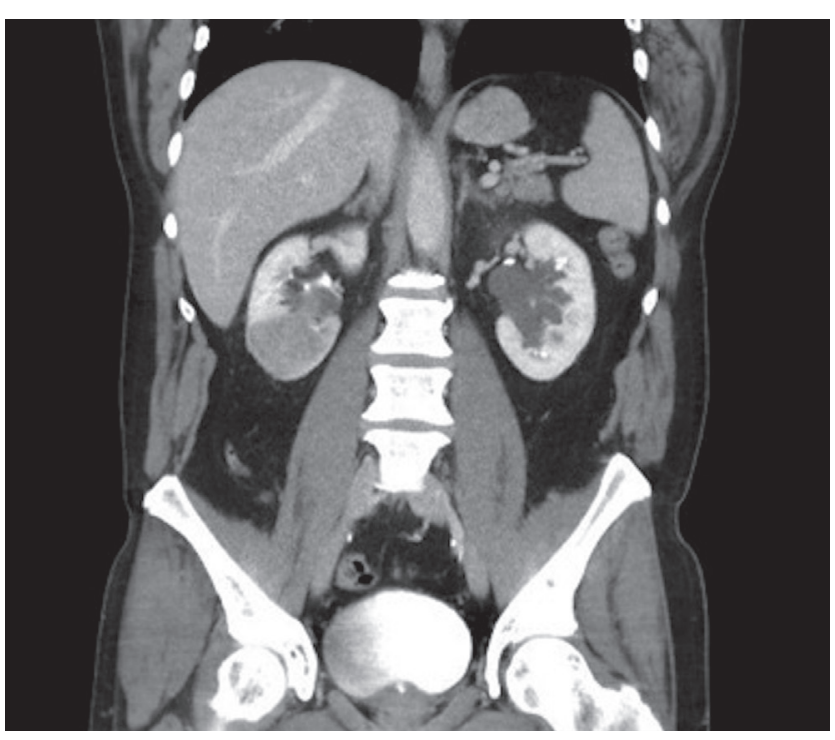

FIGURE 1 Coronal computed tomography slice showing a mass in the right kidney, with extension into the renal collecting system. 
inflammatory infiltrate. The cells co-expressed keratin and vimentin by immunohistochemistry, and were positive for PAX8, favouring a renal cell carcinoma. The differential diagnosis included unclassified renal cell carcinoma, collecting duct carcinoma, and medullary carcinoma.

Given that the pathology diagnosis was unclear on biopsy, the patient went on to have a right radical nephrectomy and retroperitoneallymph node dissection. Pathology showed a $5.5 \mathrm{~cm}$ lower pole solid mass [Figure 2(A)] involving the renal papilla, collecting system, and hilar fat. The carcinoma was forming nests, cords, and tubules, and showed a prominent neutrophilic infiltrate and desmoplastic stromal reaction [Figure 2(B)]. The tumour cells had abundant eosinophilic cytoplasm, with pleomorphic nuclei containing macronucleoli [Figure 2(C)], and they were positive for cytokeratin 7 , cytokeratin 8/18, monokeratin, vimentin, p53, and PAX8. They also showed loss of INI1. Of 23 retroperitoneal lymph nodes, 6 were positive, making this a pT3aN1 cancer.

Postoperatively, after further history, the patient reported Greek heritage and a family history of sickle cell anemia. Hemoglobin electrophoresis showed findings consistent with SCT with a hemoglobin S quantification of $38 \%$. Given the new information, a diagnosis of RMC was made.

Adjuvant chemotherapy (6 cycles of paclitaxelcarboplatin) was given. The patient was initially followed every 3-4 months with cT imaging of chest, abdomen, and pelvis; then, 24 months post-nephrectomy, the imaging frequency was decreased to every 6 months. There has been no evidence of recurrence to 38 months post-nephrectomy.

\section{REVIEW OF THE LITERATURE}

When 34 cases of RMC were first reported in 1995, the disease was described as the "seventh sickle cell nephropathy" . More recently, two large pooled reports were published. The first, by Iacovelli et al. ${ }^{2}$, describes 166 patients (19952013). The second, by Alvarez et al. ${ }^{3}$, describes 217 patients (1995-2014) and appears to include at least 154 (93\%) of the patients from the Iacovelli report. A third report, a multiinstitutional cohort, includes 52 patients treated from 2000 to 2015 at 8 institutions ${ }^{4}$. Reviewing the authorship lists, it appears that at least some of the latter patients would have also been included in the other two pooled analyses.

\section{Epidemiology}

Based on the foregoing reports and others, RMC is estimated to constitute less than $0.5 \%$ of all renal carcinomas ${ }^{5}$. It presents almost exclusively in patients with SCT, although it has also been reported in individuals with sickle cell anemia, hemoglobin S with beta-thalassemia, and hemoglobin $\mathrm{SC}^{1-5}$. It does not occur in hemoglobinopathies that do not cause red blood cell sickling.

Given the association with SCT, RMC predominantly affects patients with African heritage because of the $7 \%-10 \%$ prevalence in the African American population and up to $30 \%$ prevalence in the sub-Saharan African population ${ }^{6}$. Other populations with a high prevalence of SСт include those from Mediterranean countries (especially Greece, $1.5 \%-7.5 \%)$, the Middle East $(0.2 \%-27 \%)$, some parts of India (up to $13 \%$ ), and Latin countries $(0.2 \%-6.3 \%)^{7}$.

Renal medullary carcinoma predominantly affects adolescents and young adults ${ }^{2}$. Mean age at diagnosis is 24.3 years (range: $5-69$ years) ${ }^{3}$. The sex predominance is $2.4: 1$ male-to-female ${ }^{3}$.

\section{Presentation}

Most patients present with hematuria and flank pain; weight loss and abdominal masses are also common ${ }^{4,5}$.

The primary tumours have an average size of $6 \mathrm{~cm}$, and in most reported cases, lymph node involvement is demonstrated $^{2-4}$. Tumours are right-sided in $70 \%$ of cases $^{3,4}$. Metastatic disease was present in $80.5 \%$ of the patients in one pooled analysis and in $67.3 \%$ of those in the multiinstitutional cohort. Common sites of metastasis include lymph nodes, lung, liver, adrenal glands, and bone ${ }^{2,4}$. Brain metastasis appears to be uncommon.

Typical cT features include a central renal location, often with involvement of the renal cortex and renal sinus, an infiltrative and heterogeneous appearance, and frequent caliectasis $^{8}$. Ultrasonography, Ст imaging, and magnetic resonance imaging do not provide findings specific for RMC.

\section{Molecular Findings and Pathogenesis}

Recent studies have shown that RMC has biallelic loss of $S M A R C B 1 / I N I 1^{5,9}$. SMARCB1 is a tumour suppressor gene involved in chromatin remodelling. It is located on
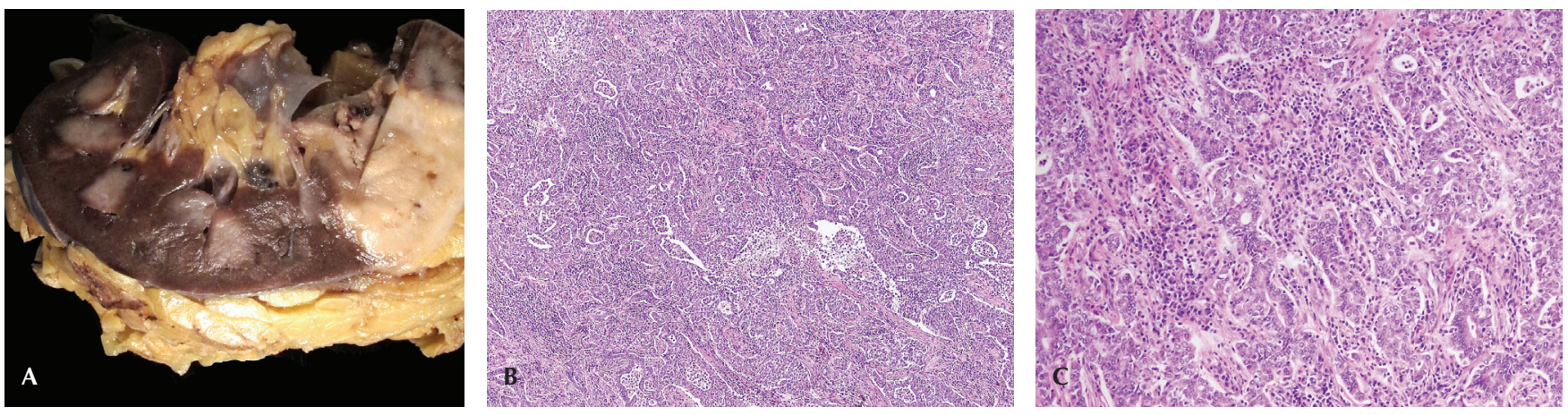

FIGURE 2 (A) The nephrectomy specimen, showing tumour involving the renal pyramids and cortex. (B) Low-power histologic view shows a tubular growth pattern, desmoplasia, and neutrophil-rich inflammatory infiltrate in the tumour. (C) Medium-power view histologic shows a high-grade adenocarcinoma appearance of the tumour cells. 
chromosome 22 at q11.2, which is a known hotspot for $d e$ novo deletions and translocations.

The exact pathogenesis of RMC is unclear, but new data and concepts are emerging. Key predisposing features thought to interact include the hypoxic and hypertonic environment in the renal medulla, the vulnerability of the $S M A R C B 1$ gene to translocations and deletions, and the sickling hemoglobinopathy ${ }^{9}$.

The hypoxic environment of the inner medulla is below the sickling threshold, leading to sickling, regional occlusion, and ischemia. That environment is thought to lead to DNA double-strand breaks in the inner medulla. Because of the hypoxia, additional error-prone repair pathways come into play, producing more translocations and deletions and potentially leading to SMARCBl inactivation.

Patients with SCT more commonly develop hematuria from the left kidney, and yet RMC occurs more frequently on the right side ${ }^{9}$. The proposed mechanism is that, because the right renal artery is longer, blood flow to the right medulla is reduced, further exacerbating the already existing microinfarctions and ischemia that predispose to $\mathrm{RMC}^{9}$.

\section{Pathology}

Renal medullary carcinoma can display a number of growth patterns, including the infiltrative high-grade adenocarcinoma morphology seen in the present case. Adenoid cystic, reticular, tubulopapillary, and microcystic patterns might also be seen. These tumours are often associated with necrosis, desmoplasia, and neutrophilic inflammatory infiltrate. Cytologic and nuclear atypia are pronounced. Immunohistochemically, the tumours are positive for PAX8 and frequently positive for cytokeratin 7 and polyclonal carcinoembryonic antigen. Loss of INI1, and acquisition of stem-cell marker ост3/4 can be helpful in making the diagnosis.

A definitive diagnosis of RMC requires confirmation that the patient has SCT or another sickling hemoglobinopathy. Morphologically and immunohistochemically identical tumours can occur without that association, and such cases are recommended to be diagnosed as "unclassified renal cell carcinoma with renal medullary phenotype" 10 .

\section{Treatment}

The RMC Working Group reviewed the data and their experience to establish standards of care ${ }^{5}$. For localized disease, the group recommends radical nephrectomy with retroperitoneal lymph node dissection. They did not make a recommendation about adjuvant therapy. In patients with metastatic disease, a good performance status, and low metastatic burden, they recommend upfront cytoreductive nephrectomy with retroperitoneal lymph node dissection, followed by systemic platin-based chemotherapy. In the review conducted by Shah et al. ${ }^{4}$, overall survival was increased in patients who underwent nephrectomy and systemic chemotherapy compared with those who underwent chemotherapy alone (16.4 months vs. 7.0 months, $p<0.001)$. The ideal management for all patients with RMC remains participation in a clinical trial.

Based on discoveries about the loss of SMARCB1/INIl in RMC, new therapies are being developed. The EZH2 protein is responsible for methylation of a specific histone involved in chromatin remodelling. An inhibitor of EZH2, tazemetostat, is currently in clinical trials.

The prognosis of patients with RMC is quite poor. Median overall survival in all comers is 13 months ${ }^{4,5}$.

\section{Long-Term Survivors}

Based on the published data, in only 7 other reported cases did patients survive with no evidence of disease for more than 24 months after diagnosis or surgery. Table I presents detailed information about the 8 long-term survivors, including our patient. With the exception of our patient, these survivors were all less than 21 years of age. In 5 patients, there was no evidence of metastases; 2 patients had lymph node involvement together with the primary;

TABLE I Reported cases of renal medullary carcinoma with no evidence of disease 24 or more months after surgery or diagnosis

\begin{tabular}{|c|c|c|c|c|c|c|}
\hline $\begin{array}{c}\text { Age } \\
\text { (years) }\end{array}$ & Sex & Heritage & Stage & $\begin{array}{l}\text { Tumour } \\
\text { size }\end{array}$ & Chemotherapy & $\begin{array}{l}\text { Survival } \\
\text { since Dx }\end{array}$ \\
\hline 8 & Male & African Brazilian & NOMO & NA & None & 8 Years \\
\hline 9 & Male & NA & $\begin{array}{c}\mathrm{N} 1 \mathrm{M} 1 \\
\text { (lymph nodes, lungs) }\end{array}$ & NA & $\begin{array}{c}\text { Metastatic: } \\
\text { gemcitabine-cisplatin-paclitaxel, } \\
\text { carboplatin-paclitaxel }\end{array}$ & $>3.5$ Years \\
\hline 13 & Female & NA & pT1N0M0 & $<2 \mathrm{~cm}$ & None & 6 Years \\
\hline 14 & Female & NA & pT1NOMO & $5 \mathrm{~cm}$ & None & 3 Years \\
\hline 19 & Male & NA & N1M0 & NA & Bortezomib & $>2.25$ Years \\
\hline 21 & Male & African American & pT1NOMO & $1.8 \mathrm{~cm}$ & None & $>2$ Years \\
\hline 47 & Male & Mediterranean & pT3aN1M0 & $5.5 \mathrm{~cm}$ & Adjuvant carboplatin-paclitaxel & $>3$ Years \\
\hline NA & Male & NA & $\mathrm{NOMO}^{\mathrm{a}}$ & NA & $\begin{array}{l}\text { Neoadjuvant carboplatin-paclitaxel } \\
\text { On metastasis: pazopanib, } \\
\text { gemcitabine-doxorubicin, } \\
\text { carboplatin-paclitaxel }\end{array}$ & 3.75 Years \\
\hline
\end{tabular}

a Developed metastatic disease after nephrectomy.

$\mathrm{Dx}=$ diagnosis; NA = not available. 
and 1 patient had metastatic disease at presentation. Of those 5 patients, 1 developed lung metastases 8 weeks after nephrectomy. All 8 patients underwent nephrectomy, and 4 received systemic treatment (3 with platin-based chemotherapy, and 1 with bortezomib, a proteasome inhibitor, on a clinical trial). There seems to be no common theme suggesting why these 8 patients had better outcomes compared with the many who often experience recurrence and die very quickly.

\section{SUMMARY}

Renal medullary carcinoma is an aggressive malignancy, and even among the few patients who present with localized disease, almost all experience recurrence, with few options for subsequent treatment. In the past, research has been limited; however, a number of recent molecular discoveries could have pathogenic and therapeutic implications. Given the increasing multicultural make-up of the Canadian population, including individuals from countries with a high prevalence of SCT, exploring for a personal or family history of hemoglobinopathy in patients with renal masses is essential.

\section{CONFLICT OF INTEREST DISCLOSURES}

We have read and understood Current Oncology's policy on disclosing conflicts of interest, and we declare that we have none.

\section{AUTHOR AFFILIATIONS}

*Faculty of Medicine, ${ }^{\dagger}$ Department of Pathology, Faculty of Medicine, ${ }^{\ddagger}$ Department of Diagnostic Radiology, Faculty of Medicine, ${ }^{\S}$ Department of Urology, Faculty of Medicine, and $\|_{\text {Division of }}$
Medical Oncology, Department of Medicine, Faculty of Medicine, Dalhousie University, Halifax, NS.

\section{REFERENCES}

1. Davis CJ Jr, Mostofi FK, Sesterhenn IA. Renal medullary carcinoma. The seventh sickle cell nephropathy. Am J Surg Pathol 1995;19:1-11.

2. Iacovelli R, Modica D, Palazzo A, Trenta P, Piesco G, Cortesi E. Clinical outcome and prognostic factors in renal medullary carcinoma: a pooled analysis from 18 years of medical literature. Can Urol Assoc J 2015;9:E172-7.

3. Alvarez O, Rodriguez MM, Jordan L, Sarnaik S. Renal medullary carcinoma and sickle cell trait: a systematic review. Pediatr Blood Cancer 2015;62:1694-9.

4. Shah AY, Karam JA, Malouf GG, et al. Management and outcomes of patients with renal medullary carcinoma: a multicentre collaborative study. BJU Int 2017;120:782-92.

5. Beckermann KE, Sharma D, Chaturvedi S, et al. Renal medullary carcinoma: establishing standards in practice. J Onc Pract 2017;7:414-21.

6. Alvarez OA. Renal medullary carcinoma: the kidney cancer that affects individuals with sickle cell trait and disease.JOnc Pract 2017;13:424-5.

7. Vichinsky EP. Sickle cell trait [Web article]. Waltham, MA: UpToDate, Wolters Kluwer Health; 2018. [Current version available at: https://www.uptodate.com/contents/sicklecell-trait (paid subscription required); cited 17 January 2019]

8. Greco F, Faiella E, Santucci D, et al. Imaging of renal medullary carcinoma. J Kidney Cancer VHL 2017;4:1-7.

9. Msaouel P, Tannir NM, Walker CL. A model linking sickle cell hemoglobinopathies and SMARCB1 loss in renal medullary carcinoma. Clin Cancer Res 2018;24:2044-9.

10. Amin MB, Smith SC, Agaimy A, et al. Collecting duct carcinoma versus renal medullary carcinoma: an appeal for nosologic and biological clarity. Am J Surg Pathol 2014;38:871-4. 